VOL. II (1974), 43-46.

\title{
Defining relations for the \\ Held-Higman-Thompson simple group
}

\section{John J. Cannon and George Havas}

A set of defining relations for the Held-Higman-Thompson simple group of order 4030387200 is given.

In [3], Held gives properties of a possible new simple group $H$ of order $2^{10} \cdot 3^{3} \cdot 5^{2} \cdot 7^{3} \cdot 17$, whose character table was constructed by Thompson. This simple group is characterised as having the same centralizer of an involution as $M_{24}$ and $L_{5}(2)$. Unpublished work of Graham Higman and Mckay established that the smallest degree permutation representation of $H$ is on 2058 letters. The stabilizer of a point of this representation is $S p_{4}(4)$, extended by an automorphism of order 2 . Let $K$ be a subgroup of $H$ isomorphic to $S p_{4}(4)$ and $K^{*}$ a subgroup isomorphic to $S p_{4}(4)$ extended by the automorphism.

Using the fact that $\mathrm{H}$ contains $S p_{4}(4)$, Higman constructed defining relations for a group $H^{*}$ that contains $H$ as a subgroup of index 2 . Thus the existence of $H$ was established. Higman's relations for $H^{*}$ are (McKay [5]):

$$
\begin{gathered}
a^{2}=b^{3}=c^{2}=d^{4}=e^{2}=\left(a d^{2}\right)^{2}=\left(c d^{2}\right)^{2}=\left(b d^{2}\right)^{2}=(a d)^{8}=\left(b d b^{-1} d\right)^{2}= \\
{[a, b]=a d b d a d b^{-1} d b^{-1}=[c, b]=\left(d^{-1} b d c\right)^{3}=\left((a d)^{3} c d\right)^{2}=(a c)^{3}=} \\
(a d b d c d b d)^{5}=(c a d c d a)^{2}=(c d a d)^{4}=(c d)^{4} a d c a d c d a d a=(d e)^{2}= \\
{[c, e]=\left(a d^{2} e\right)^{3}=(a d a d a e)^{3}=b^{-1} d b d e b d b d e=1 .}
\end{gathered}
$$

Received 20 March 1974. This work was supported by a grant from the Australian Research Grants Committee. 
Using a program which determines all subgroups of a finitely presented group up to a specified index (Lepique [4]), we found a unique subgroup of index 2 , generated by

$$
a, b, c, d^{2}, e, d^{-1} a d, d^{-1} c d
$$

which can only be $H$. Further investigation revealed that the first six of these words suffice to generate $H$. However, contrary to Higman's earlier belief, the first five words generate a proper subgroup of $H$.

The next step was to apply a Reidemeister-Schreier program [2] to present this subgroup. This program constructed a seven generator, fortyfive relator presentation for $H$. Further simplification of this presentation by hand resulted in the following six generator, thirty-seven relator presentation:

$$
\begin{aligned}
u^{2}=v^{2}= & w^{2}=x^{2}=y^{2}=z^{3}=(v w)^{2}=(w y)^{2}=[x, z]=[y, z]=(u v)^{3}= \\
& (x y)^{3}=(w x)^{3}=(v z)^{3}=(u v u y)^{2}=(u z)^{2}\left(u z^{-1}\right)^{2}=(v x y x)^{2}=(u x)^{4}= \\
& (u x u y)^{2}=(u x v x)^{2}=(u y)^{4}=(v x)^{4}=(u z u x)^{2}=u z u z^{-1} w u z v w= \\
& u v u z u z v z^{-1} u z^{-1}=u v x v y v y x u y=(u w u x)^{2} w r w x=(u x w x)^{3}=(u w)^{6}= \\
& (u z)^{6}=\left(v(u w)^{3}\right)^{2}=\left(x(u w)^{3}\right)^{2}=\left(y(u w)^{3}\right)^{2}=\left(z(u w)^{3}\right)^{2}= \\
& \left.\left(u y z^{-1} u z^{-1}\right)^{3}=\left(u z v z^{-1}\right)^{5}=(\text { xuzuyuz })^{-1} u\right)^{5}=1 .
\end{aligned}
$$

The relationship between these generators and the generators for $H^{*}$ is as follows:

$$
\begin{aligned}
& u=a ; \quad x=d a d^{-1} ; \\
& v=c ; \quad y=d c d^{-1} ; \\
& w=e ; z=b .
\end{aligned}
$$

This presentation involves redundant generators. Indeed $H$ is generated by the two elements $x$ and $u y x z^{-1} w z^{-1}$.

We now proceed to identify subgroups $K$ and $K^{*}$. Using the socalled random coincidence procedure in conjunction with the Todd-Coxeter algorithm (Cannon and Havas [1]) it was found that

$$
K^{*}=\left\langle z^{-1} y v z^{-1} x v z, \text { wravraw }\right\rangle
$$


and

$$
K=\left\langle u, v,(u z)^{2}, z^{-1} y v z^{-1} x v z\right) \text {. }
$$

Other subgroups located using this procedure were

$$
L=\left\langle u, v,(u z)^{2}, z^{-1} y v x w v z x, \text { wrouraw }\right\rangle
$$

of index 8330 , and

$$
M=\left\langle u, v,(u z)^{2}, z^{-1} y v x w v z x\right\rangle
$$

of index 16600 .

It is probable that $L$ and $M$ are related to the centralizer of the involution $t$ in $H$ (see [3]).

Enumerating cosets of $K^{*}$ in $H$ gives the permutation representation of $H$ on 2058 letters. This permutation group was generated using the Sydney group theory system, GROUP, and found to have correct order of 4030387200 . Further application of these programs showed that $K^{*}$ has five orbits of lengths $1,136,136,425$ and 1360 . Thus $H$ is a rank 5 group.

\section{References}

[1] John J. Cannon and George Havas, "Applications of the Todd-Coxeter algorithm", (Computer-aided Mathematics Project, Technical Report, 6. Department of Pure Mathematics, University of Sydney, Syaney, 1974).

[2] George Havas, "A Reidemeister-Schreier program", Proc. Second Intermat. Conf. Theory of Groups, Canberra, 1973, 347-356 (Lecture Notes in Mathematics, 372. Springer-Verlag, Berlin, Heidelberg, New York, 1974).

[3] Dieter Held, "The simple groups related to $M_{24}$ ", J. Algebra 13 (1969), 253-296.

[4] Evelyn Lepique, "Ein Programm zur Berechnung von Untergruppen von gegebenem Index in endlich präsentierten Gruppen" (Diplomarbeit, Rheinisch-Westfälische Technische Hochschule, Aachen, 1972). 
[5] John McKay, "Computing with finite simple groups", Proc. Second Internat. Conf. Theory of Groups, Canberra 1973, $448-452$ (Lecture Notes in Mathematics, 372. Springer-Verlag, Berlin, Heidelberg, New York, 1974).

Department of Pure Mathematics,

University of Sydney,

Sydney,

New South Wales;

School of Information Sciences,

Canberra College of Advanced Education,

Canberra, ACT. 